\title{
Orbital Lymphoma Mimicking Lacrimal Gland Pleomorphic Adenoma
}

\author{
Diego Strianese Andrea Elefante Francesco Matarazzo Angela Panico \\ Mariantonia Ferrara Fausto Tranfa \\ Department of Ophthalmological Sciences, University 'Federico II' of Naples, Naples, Italy
}

\section{Key Words}

Orbital lymphoma · Pleomorphic adenoma · Lacrimal gland

\begin{abstract}
Purpose: To describe the case of a patient affected by orbital lymphoma mimicking pleomorphic adenoma of the lacrimal gland. Methods: This was a retrospective case report. Results: We present the case of a patient with 15-year history of slowly progressive left proptosis and inferomedial bulbar dislocation who had the presumptive diagnosis of lacrimal gland pleomorphic adenoma based on clinical and radiological features. The patient underwent lateral orbitotomy and lacrimal gland excision. Postoperative histological features were consistent with low-grade B-cell non-Hodgkin lymphoma. Conclusion: The accepted clinico-radiological criteria used for the diagnosis of lacrimal gland fossa lesions might have a certain false-positive rate, even in recent years. The initial surgical approach with the appropriate choice between fine-needle aspiration biopsies, intraoperative biopsies and lacrimal gland excisions might be a challenge.

(C) 2013 S. Karger AG, Basel
\end{abstract}

\section{Introduction}

Lymphoma is one of the more frequent orbital malignant tumors, accounting for $11 \%$ of all orbital tumors and for $55 \%$ of malignant tumors [1]. It is commonly thought that clinical and radiological features along with the clinical history make the presumptive diagnosis of orbital lymphoma easily accessible. However, differential diagnosis with specific or idiopathic orbital inflammation, benign or malignant tumors, especially if located in the 
lacrimal fossa, may be challenging [2-5]. We present the case of a patient with orbital lymphoma mimicking lacrimal gland pleomorphic adenoma (LGPA).

\section{Materials and Methods}

This study was carried out according to the Declaration of Helsinki on medical protocol and ethics. A 58-year-old female patient, following an episode of fainting, underwent a computed tomography (CT) scan of the brain, which did not show any traumatic lesion, but incidentally revealed a left orbital mass involving the lacrimal gland, homogeneously hyperintense modeling of the lateral and superior recti eye muscles and the adjacent orbital bone. Clinical examination showed a $25-\mathrm{mm}$ exophthalmos on the Hertel readings with downwards and medial dislocation of the eye bulb, $2 \mathrm{~mm}$ ptosis of the left eyelid (MRD of 2 $\mathrm{mm}$ ), BCVA of 20/20 in both eyes, no diplopia, no gaze restriction [6], intraocular pressure of $16 \mathrm{~mm} \mathrm{Hg}$ in both eyes, and no remarkable findings at the fundus examination. Clinical history and the review of the patient's pictures taken in the past revealed a 15-year history of slowly progressive left proptosis (fig. 1). She actually was never aware of it. Magnetic resonance imaging (MRI) showed an oval, well-defined mass, which appeared slightly hyperintense on both T1-weighted and T2-weighted imaging (fig. 2) with marked enhancement of gadolinium on contrast-enhanced MR images (fig. 3). These radiological findings suggested a benign tumor of the lacrimal gland. No further distinguishing clinical or blood test features were found (including general diseases frequently associated with orbital lymphoma, such as hepatitis $\mathrm{C}$ infection [7]). The patient underwent lateral orbitotomy to remove the lacrimal gland including the whole mass. During surgery, the orbital mass appeared as a greyish bilobular mass, easy to isolate from near tissues. Pathological findings revealed a low-grade B-cell non-Hodgkin lymphoma arising from the lacrimal gland. The patient underwent postoperative radiotherapy [8] with favorable outcome and no recurrences in a 3-year follow-up period.

\section{Discussion}

Orbital lymphoma appearing in the lacrimal gland region accounts for about $11 \%$ of all orbital lymphomas [1]. Clinical history usually includes onset of proptosis, which can be from fairly slow to sudden [9]. The patient was incidentally found to have a lacrimal gland tumor, and only after an accurate review of her previous pictures, the slowly progressive proptosis was revealed. The patient was never aware of her left exophthalmos, an entity that might be suspicious of a benign lacrimal gland tumor, but it is an unusual clinical presentation of an orbital lymphoma [9]. An orbital lymphoma usually manifests as a diffuse, solid, enhancing mass, characterized by molding around the globe on imaging studies, reflecting the irregular infiltration of orbital structures, and only appears to be defined in $20 \%$ of patients [10]. The recent literature [10] indicates that orbital lymphoma appears to be isointense compared to extraocular muscles on T1-weighted and T2-weighted images. This is in contrast to earlier studies showing how orbital lymphoma appears to be isointense compared to extraocular muscles on T1-weighted images and hyperintense on T2-weighted imaging [11], but this may explain one of the most predictive features for the malignancyisointense mass on T2-weighted imaging $[12,13]$. Variable enhancement has been reported after contrast administration $[10,11]$. In our case, CT and MRI reports were nonspecific for lymphoma and had some similarities with LGPA. Particularly, on the left lacrimal gland, an 
oval, well-defined mass appeared with some aspects of nodularity, slightly hyperintense on both T1- and T2-weighted imaging. Those features were more suggestive of a benign lesion of the lacrimal gland, such as pleomorphic adenoma. On MR and CT imaging, benign mixed tumors of the lacrimal gland are seen as round to oval lesions rather than as compressed, oblong and 'almond-shaped' lesions. The masses are generally well circumscribed. Nodularity and infiltrative appearance on MR or CT imaging should raise the question of the differential diagnosis between carcinomas and pleomorphic adenomas, although at times, a benign mixed tumor may show marked nodularity and an infiltrative pattern. Calcification is more common in carcinomas; however, it may be present in adenomas as well. LGPAs on MR imaging demonstrate long T1 and T2 signal characteristics. These tumors may be heterogeneous on T2-weighted MR images or may display a diffuse nodular pattern. They show moderate to marked enhancement on contrast-enhanced MR images and CT scans [13]. Since the pathological diagnosis of epithelial lacrimal gland tumors is a crucial issue in terms of prognosis, it is mandatory to perform the excisional biopsy [14, 15]. For this reason, our patient underwent lacrimal gland excision through lateral orbitotomy. Considering the final pathological result of lymphoma, the procedure we performed could appear more invasive than necessary. In fact, for orbital lymphoma, a simple incisional biopsy is suggested. However, it should be considered that in a study [2] including 14 patients who underwent lacrimal gland excision because of a preoperative diagnosis of LGPA, the postoperative histology revealed lymphoma in 4 patients. The authors found that $22.6 \%$ of suspected LGPA were misdiagnosed based on clinico-radiological criteria.

\section{Conclusions}

This report demonstrates that the accepted clinico-radiological criteria used for the diagnosis of lacrimal gland fossa lesions have a high false-positive rate, even in experienced hands and in the modern era (as already reported in the literature [2]). The choice between fine-needle aspiration biopsies, intraoperative biopsies and lacrimal gland excision for the initial appropriate management of these lesions might still be a challenge.

\section{Disclosure Statement}

The authors declare no conflict of interest. No financial support was received for this submission.

\section{References}

1 Bonavolontà G, Strianese D, Grassi P, Comune C, Tranfa F, Uccello G, Iuliano A: An analysis of 2,480 spaceoccupying lesions of the orbit from 1976 to 2011. Ophthal Plast Reconstr Surg 2013;29:79-86.

2 Prabhakaran VC, Cannon PS, McNab A, Davis G, O’Donnell B, Dolman PJ, Ghabrial R, Selva D: Lesions mimicking lacrimal gland pleomorphic adenoma. Br J Ophthalmol 2010;94:1509-1512.

3 Bonavolonta P, Strianese D, Vecchione ML, Staibano S: A challenging case of primary orbital mesenchymal chondrosarcoma. Orbit 2010;29:281-283.

4 Strianese D, Piscopo R, Elefante A, Napoli M, Comune C, Baronissi I, Liuzzi R,Ferrara M, D'alessandro A, Ruggiero $\mathrm{P}$, Napolitano $\mathrm{P}$, Grassi P, Iuliano A, RussoC, Brunetti A, Bonavolontà G: Unilateral proptosis in thyroid eye disease with subsequent contralateral involvement: retrospective follow-up study. BMC Ophthalmol. 2013;13:21. 
Strianese et al.: Orbital Lymphoma Mimicking Lacrimal Gland Pleomorphic Adenoma

5 Madge SN, Tumuluri K, Strianese D, Bonavolonta P, Wilcsek G, Dodd TJ, Selva D: Primary orbital liposarcoma. Ophthalmology 2010;117:606-614.

-6 Dolman PJ, Cahill K, Czyz CN, Douglas RS, Elner VM, Feldon S, Kazim M,Lucarelli M, Sivak-Collcott J, Stacey AW, Strianese D, Uddin J: Reliability of estimating ductions in thyroid eye disease: an International Thyroid Eye Disease Society multicenter study. Ophthalmology 2012;119:382-389.

-7 Strianese D, Tranfa F, Finelli M, De Renzo A, Staibano S, Schiemer R, Cardone D, Pacelli R, Perna F, Mascolo M, De Rosa G, Bonavolontà G: Hepatitis C virus infection in ocular adnexal lymphomas. Arch Ophthalmol 2010;128:1295-1299.

$>8$ De Cicco L, Cella L, Liuzzi R, Solla R, Farella A, Punzo G, Tranfa F, Strianese D, Conson M, Bonavolontà G, Salvatore M, Pacelli R: Radiation therapy in primary orbital lymphoma: a single institution retrospective analysis. Radiat Oncol 2009;4:60.

-9 Tranfa F, Di Matteo G, Strianese D, Forte R, Bonavolontà G: Primary orbital lymphoma. Orbit 2001;20:119124.

-10 Xian J, Zhang Z, Wang Z, Li J, Yang B, Man F, Chang Q, Zhang Y: Value of MR imaging in the differentiation of benign and malignant orbital tumors in adults. Eur Radiol 2010;20:1692-1702.

$\checkmark 11$ Polito E, Galieni P, Leccisotti A: Clinical and radiological presentation of 95 orbital lymphoid tumors. Graefes Arch Clin Exp Ophthalmol 1996;234:504-509.

12 Mafee MF, Edward DP, Koeller KK, Dorodi S: Lacrimal gland tumors and simulating lesions. Clinicopathologic and MR imaging features. Radiol Clin North Am 1999;37:219-239.

-13 Iuliano A, Strianese D, Uccello G, Diplomatico A, Tebaldi S, Bonavolontà G: Risk factors for orbital exenteration in periocular basal cell carcinoma. Am J Ophthalmol 2012;153:238-241.

14 Weis E, Rootman J, Joly TJ, Berean KW, Al-Katan HM, Pasternak S, Bonavolontà G, Strianese D, Saeed P, Feldman KA, Vangveeravong S, Lapointe JS, White VA: Epithelial lacrimal gland tumors: pathologic classification and current understanding. Arch Ophthalmol 2009;127:1016-1028.

15 Strianese D, Baldi G, Staibano S, Baldi A, De Rosa G, Tranfa F, Bonavolontà G: Expression of apoptosis-related markers in malignant epithelial tumours of the lacrimal gland and their relation to clinical outcome. Br J Ophthalmol 2007;91:1239-1243.

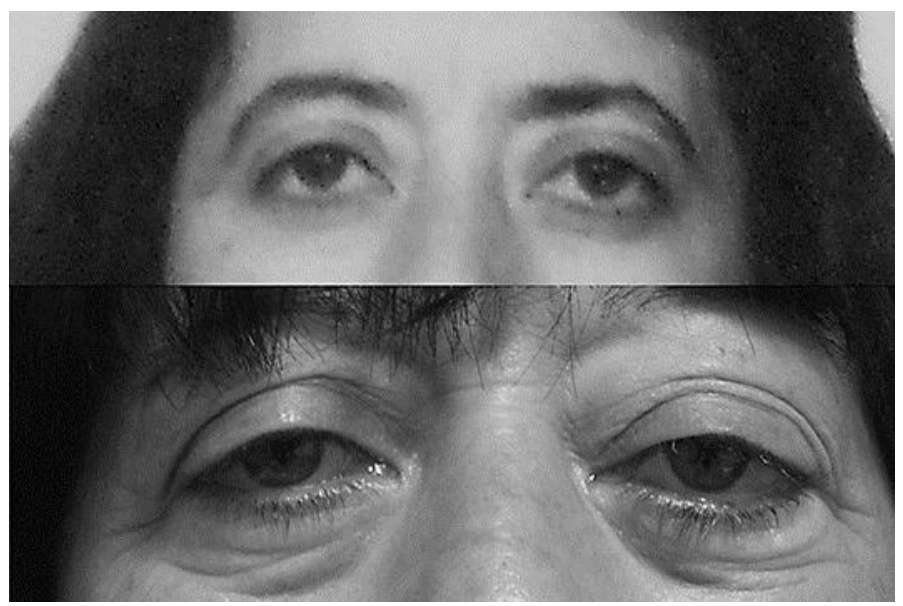

Fig. 1. Slowly progressive left proptosis: before and after 15 years. 
Case Reports in

Ophthalmology

\begin{tabular}{l|l}
\hline Case Rep Ophthalmol 2013;4:109-113 \\
\hline DOI: 10.1159/000354963 & $\begin{array}{l}\text { ○ 2013 S. Karger AG, Basel } \\
\text { www.karger.com/cop }\end{array}$ \\
\hline
\end{tabular}

Strianese et al.: Orbital Lymphoma Mimicking Lacrimal Gland Pleomorphic Adenoma

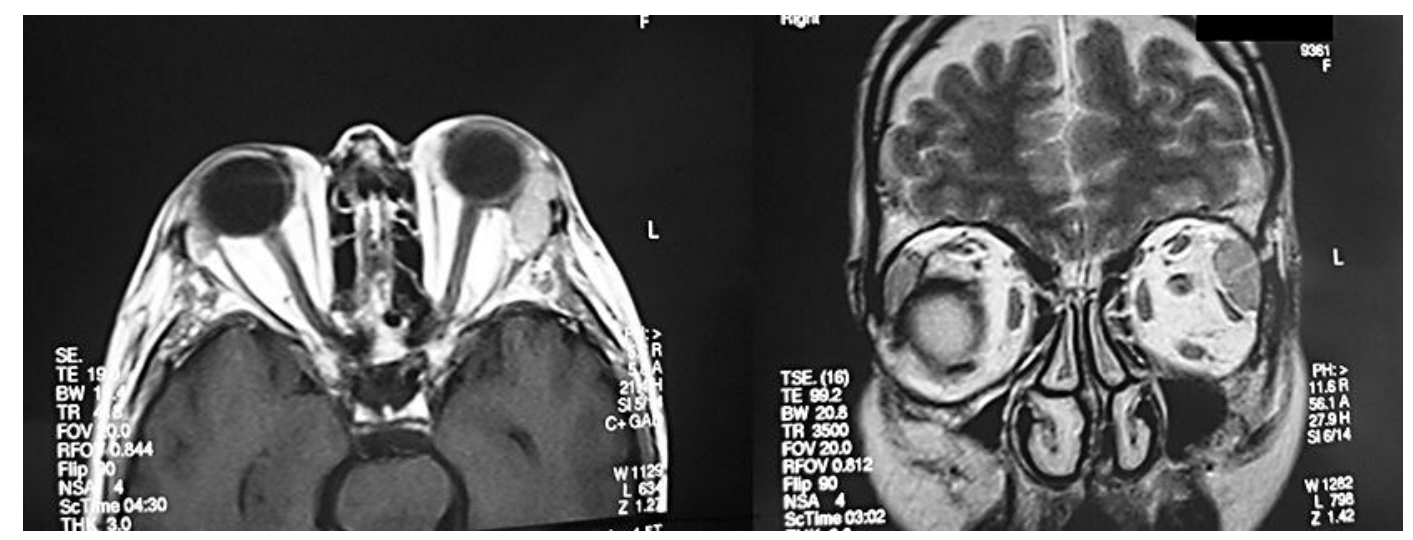

Fig. 2. The lesion appears slightly hyperintense on both T1-weighted images and T2-weighted imaging.

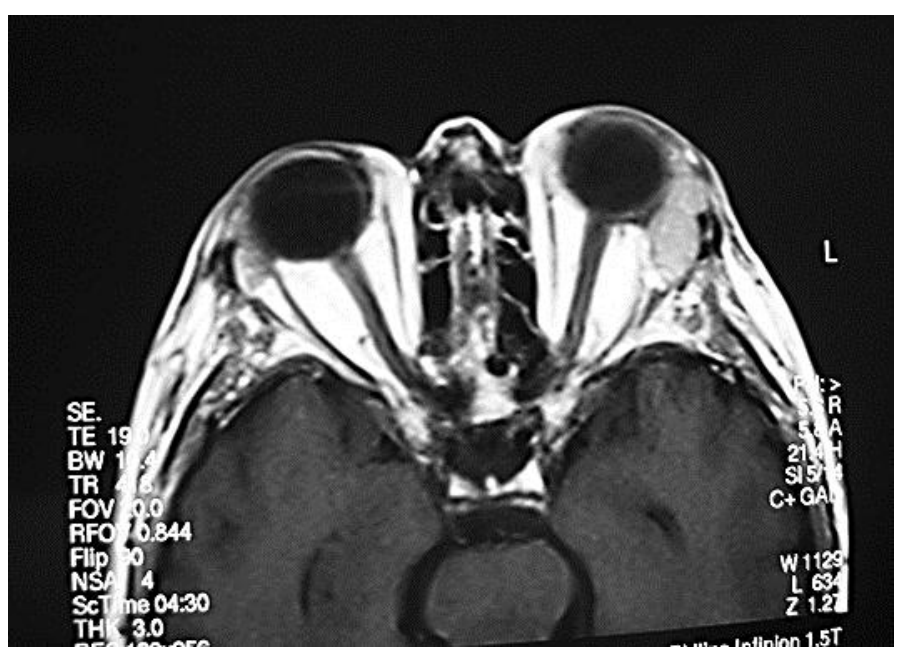

Fig. 3. On contrast-enhanced SE T1 FAT-SAT MR images, the lesion shows marked and homogeneous enhancement of gadolinium. 